\title{
FACT: A Dutch Version of ACT
}

\author{
J. Remmers van Veldhuizen, Psychiatrist
}

\begin{abstract}
Assertive Community Treatment (ACT) is a well-defined service delivery model for the care and treatment of the most severely mentally ill in the community. We have opted for a Dutch version named 'Function' ACT or FACT. In a FACT team, ACT is one of the functions that the team can perform. For more stable long-term patients FACT provides coordinated multidisciplinary treatment and care by individual case management. Unstable patients at risk of relapse are followed with assertive outreach care by the same team, working with a shared caseload for this subgroup. This article describes the service model and everyday practice in FACT.
\end{abstract}

KEY WORDS: Assertive Community Treatment (ACT); FACT; community care systems; social inclusion

Assertive Community Treatment (ACT) is a well-defined service delivery model for the care and treatment of the most severely mentally ill people (SMI) in the community (Stein, 1990; Stein \& Santos, 1998; Test \& Stein, 1978). An ACT team is a multidisciplinary team (including case managers, a psychiatrist, a psychologist and a substance abuse counselor), which provides assertive outreaching intensive care and treatment to a group of up to a hundred SMI. It is an evidence-based intervention (Bond, Drake, Mueser, \& Latimer, 2001; Marshall \& Lockwood, 2002). ACT teams work with a shared caseload, cover 24 hours a day and function as gatekeepers to the psychiatric wards. Program fidelity to ACT can be measured by the Dartmouth ACT Scale (Bond \& Salyers, 2004; Teague, Bond, \& Drake, 1998; Teague,

J. Remmers van Veldhuizen, psychiatrist, is director of care innovation at the Noord-Holland Noord GGZ (Mental Health Care Service).

Address correspondence to J. Remmers van Veldhuizen, GGZ Noord-Holland Noord, P. O. Box 18, Heiloo, 1050 BA, The Netherlands; e-mail: remmersvv@hotmail.com. 
Dartmouth Assertive Community Treatment Scale). In some areas in the Netherlands ACT teams have been set up, but more regions have opted for a Dutch version named FACT. ${ }^{1}$

FACT is a rehabilitation-oriented clinical case management model, which is based on the ACT model but is more flexible and able to serve a broader range of clients with severe mental illness. FACT offers the original ACT as one of several treatment or care models. The FACT team is a case management team with partly an individual approach and partly a team approach; the approach varies from patient to patient, depending on the patient's needs. For more stable long-term patients FACT provides coordinated multidisciplinary treatment and care by individual case management. Unstable patients at risk of relapse, neglect and readmission are provided with intensive assertive outreach care by the same team, working with a shared caseload for this subgroup.

This article describes the development of the FACT model, the service model and everyday practice in FACT. We discuss the advantages of FACT as compared with standard ACT and future challenges in the Dutch situation.

\section{MENTAL HEATH CARE IN THE NETHERLANDS}

The Dutch mental health care system is relatively rich and to a considerable extent still hospital-based. In contrast with other European countries, the United States and Australia, only a moderate degree of deinstitutionalization has taken place (Wing et al., 1998). In 2005 there were 12,000 longstay beds for a population of 16 million.

Acute and cure-focused psychiatry is concentrated in Multi-functional Entities (MFE): small 24-48 bed hospital units with a wide range of outpatient functions, preferably located in or near the general hospital. They also provide acute partial hospitalization with outreach home care for the acutely mentally ill, with very short hospital admissions if necessary. This possibility is referred to as the 'bed on request' (BOR); an important aspect is that the patient does

\footnotetext{
1 The $\mathrm{F}$ in the Dutch acronym FACT originally stood for 'function'. The idea behind the term is that the FACT team functions in different ways: for the $20 \%$ group the FACT team functions in exactly the same way as an ACT team, but it also has many other functions. However, in English we would like to have the $\mathrm{F}$ also stand for 'Flexible'. We hope that not too much confusion will be caused by the use of the same acronym, FACT, in America to refer to forensic ACT teams.
} 
not have to go through a entire formal admission procedure, but is admitted for one or two nights only, after which outpatient care continues (Priebe, Huxley, Knight, \& Evans, 1999). This proved to be a feasible alternative to acute care in a psychiatric hospital (Ravelli, 2005).

In the 1980s a mental health care system was developed in the Netherlands, which is known as 'transmural care': a term, which alludes to the concept that care continues beyond the walls of the institutions. This system, a flexible combination of outreach, outpatient departments, day treatment and hospital services with continuity of treatment and care, has become an important standard in the Netherlands. Twenty-four-hours-a day, 7-days-a-week crisis services have been organized in every region.

In the Netherlands the longstay wards were downsized slowly over the last 20 years. However, the needs of long-term patients in the community grew more rapidly than could be accounted for by the downsizing of the wards. As in other countries we saw increasing problems with homelessness and dual diagnosis (a serious psychiatric disorder combined with a serious addiction problem). Many projects were set up: psychiatric home care and brokerage or individual case management were the most popular forms, but most of these programs worked with individual caseloads (Veldhuizen, Wiersma, \& Ram, 1988). The majority of these programs did not reach the patients in the greatest need: the homeless, and patients with dual diagnosis and forensic problems. Meanwhile the population of psychiatric patients in the penal system and in the forensic psychiatric system had been growing rapidly since 1995 (Kluiter, 1997).

In 1994 a new mental health act came into force. This act-the Psychiatric Hospitals Compulsory Admission Act-was a reaction to the paternalistic institutions and the abuses in psychiatry of the 1950s and 1960s. Thresholds for voluntary and involuntary admission were raised. Patients committed to hospital could be treated against their will only in the event of 'severe danger in the hospital'. Problems related to people with severe mental illness grew much more rapidly than the solutions. Politicians and municipalities expressed their discontent with mental health care. At the turn of the century this whole situation led to what could be termed a paralysis of care (Henselmans, Kok, Nammensma, \& van Berkel, 1991). A growing sense of urgency for change coincided with an international spate of publications about ACT, which seemed to offer a solution. 


\section{DEVELOPMENT OF THE FACT MODEL}

Standard ACT focuses on the most severely ill psychiatric patients living in the community: those at risk of relapse and hospital readmission. Housing and medication are often unstable and dual diagnosis is common. Bond estimates that this target group constitutes $20 \%$ of the long-term mentally ill persons in a population or $.1 \%$ of the total population (Bond et al., 2005). The Cochrane study (Marshall \& Lockwood, 2002) recommends ACT for this most unstable sub-group of patients. ACT has proved effective in linking these patients to the service and in reducing bed days.

There is less evidence-based literature about the larger$80 \%$-group of more stable long-term mentally ill patients living in the community. In our region, until 2002 most of these patients were served by case management teams according to Harris's model (Veldhuizen, 1998). In some cases the psychiatrist was a member of the team, but in other cases the teams had to link the patients to psychiatrists working elsewhere in the service. The transmural care system already ensured continuity of care in the event of hospitalization and 24-hour crisis service coverage.

This system worked for many patients, but if patients became unstable and needed more support than once or twice a week, the case managers ran into problems with their caseload. As a result, too many patients were readmitted too frequently and other patients could not be discharged from the hospital. There were problems with patients with dual diagnosis. Because the teams did not include psychiatrists, psychologists and drug abuse specialists, there were problems with implementing evidence-based medical treatment and multidisciplinary guidelines.

The ACT model seemed likely to provide solutions to our problems. But in smaller and rural areas it was impossible to organize fullyimplemented ACT teams, because of the lower numbers of patients with severe mental illness in those areas. Nor did we opt for one specialized ACT team to cover several rural regions with their own generic services. According to Burns and Fiorritti (2001), one ACT team for more rural areas creates an 'uncomfortable situation', because it leads to problems regarding continuity of care and involves too much travel time. One of our priorities was to ensure regional continuity of care, and we certainly did not want ACT team to spend too much time traveling. We also questioned whether there really was 
an absolute distinction between the $20 \%$ and the $80 \%$ group. Are they separate groups, or do patients sometimes belong to one group and sometimes to the other, depending on the thresholds of the system? We suspected that there was a great deal of exchange between the two groups.

The multidisciplinary composition of the team is an important criterion for ACT. This criterion seemed important not only for the $20 \%$ group, but for the $80 \%$ group as well: they too need evidence-based treatment with medication, medication management, psycho-education, family interventions and cognitive behavioral therapy given by psychiatrists, psychologists and social workers. And they too are in need of supported employment or service by a professional with knowledge about addictions and dual diagnosis.

Another consideration had to do with recovery and rehabilitation-processes, which take time and are more relevant to patients in the $80 \%$ group than to the $20 \%$ group, who are primarily struggling to survive crisis and postpone readmission. There is a consensus in the literature that rehabilitation and recovery are most likely to be successful when there is a stable, 1-to-1 relationship between client and case manager. But the processes of rehabilitation and recovery are sometimes disrupted by a recurring psychosis, and at that point the patient enters the $20 \%$ group. We therefore thought it was necessary to ensure continuity of rehabilitation for the $20 \%$ group as well.

We concluded that the differences between the two groups pertained only to the intensity of care and treatment at a particular point in time and did not have consequences for the composition and attitude of the teams. We therefore opted to ensure regional continuity of treatment, care and rehabilitation by creating teams, which would focus on both the $20 \%$ group and the $80 \%$ group. We decided to incorporate ACT as one function in multi-functional regional case management teams: the 'FACT' teams.

\section{STARTING THE FACT MODEL}

In the North Holland region (north of Amsterdam) we decided in 2002 to set up regional FACT teams to serve $100 \%$ of long-term psychiatric patients living in the community. Each FACT team was able to provide two types of care: 
- individual case management by a multidisciplinary team for the $80 \%$ group

- shared case management and assertive outreach for the $20 \%$ group

We implemented the fidelity criteria for the multidisciplinary ACT teams by adding a psychiatrist, a part-time psychologist, an IPS (Individual Placement and Support) worker and a specialist in drug abuse. The teams' formal task was to give coordinated care, evidencebased medical treatment and recovery-oriented rehabilitation. Each FACT team was to work in one particular region with approximately 50,000 inhabitants and provide services for 200-220 long-term psychiatric patients. The FACT model was first implemented in four teams in a region with 225,000 inhabitants.

\section{HOW FACT WORKS}

Patients in the $80 \%$ group are provided with recovery-oriented individual case management. The case manager visits the patients $2-4$ times a month at their homes and is responsible for the individual care and treatment plan, which is renewed at least once a year and is formulated in a way that patients and their families can understand. Goals for rehabilitation and the organization of social support and or work are included. Part of a treatment plan is the so-called 'crisis plan', with early warning symptoms and concrete arrangements for 'intensification of care' if necessary. Regular (non-crisis) appointments with the psychiatrist (medication management, evaluation) and the psychologist (psycho-education, cognitive behavior therapy) take place at the FACT center. Family interventions and supported employment may be added to this treatment plan.

Care for the $20 \%$ group is organized around the team's whiteboard. If a patient is at risk of readmission or crisis, the case manager puts the name of the patient on the whiteboard. At that point the patient is admitted to ACT and will be served by assertive outreach. For these patients, the FACT team works on the principle of shared caseload. From the first day a patient's name appears on the whiteboard, a standard procedure starts: the patient must be seen by the psychiatrist within two days (if necessary at the patient's own home), the crisis plan is updated and set in motion. The case manager informs the patient (and if necessary the family) that more intensive care will be organized 
and that colleagues from the FACT team will work together to prevent readmission and to shorten the crisis.

The whiteboard is reviewed daily in a team meeting, in the morning or at lunchtime. The 15-30 patients on the whiteboard are discussed, just as in the routine of ACT teams. Appointments for home visits and other actions are made. Care for these patients is based on a shared caseload, and if necessary all the team members will visit a patient at home. Often, after three or more weeks, the crisis or risk of relapse of psychosis starts to decrease. In the following phase the patient's name remains on the whiteboard, but as much as possible the case manager maintains contact, and gradually the other team members step back. If the situation is stable and individual case management seems to be sufficient, the patient's name is removed from the whiteboard. The crisis plan is updated for a following episode and care as usual continues.

An average FACT team serves 200-220 patients and consists of 7-8 FTE case managers (psychiatric nurses, psychiatric community nurses, social workers, substance abuse counselors), .8 psychiatrist, .6 psychologist and .5 Supported Employment Worker. In the future it will also include a former patient as a coworker. Every team has about 50 clients who are very stable and need little contact. Our case managers thus have caseloads of 20 patients receiving regular or more intensive care. Some teams assess their patients annually with the help of standard instruments, the Health of the Nation Outcome Scale (HoNOS, Wing et al., 1998) and Manchester Short Assessment of Quality of Life (MANSA, Priebe et al., 1999), which give an indication of unmet needs on a personal level. The results are aggregated in order to evaluate the teams. The information about unmet needs is used by the case managers in drawing up care and treatment plans with their patients.

\section{FURTHER DEVELOPMENTS AND RESULTS}

We have found that in $80-90 \%$ of cases a client's need for more intensive services is temporary (for a few weeks or months) rather than permanent. Nevertheless, we do have some patients who remain on the whiteboard for a very long time. These are people whose illness is so severe that they can only live outside the hospital with daily intensive support. After 2 years it became clear that at any one point not $20 \%$ but only $10-15 \%$ of patients needed to be put on the whiteboard for ACT service with assertive outreach and shared caseload. After the FACT teams were set up, we saw a gradual decrease in admission days and an increase in admissions. 
The whiteboard includes specific sections with the names of patients who are at risk of dropout, patients with whom the team have recently lost contact, and patients who are hospitalized or in jail. Multidisciplinary collaboration between case managers and psychiatrists facilitates the use of leverage (Weeghel et al., 2005), which is important in improving adherence to psychiatric treatment. Team members can play different roles, with different degrees of intrusiveness: for example one member can implement forced treatment, while another can maintain a more neutral or closer relationship with the same patient, so that the patient does not drop out of care.

The introduction of addiction workers into the FACT teams began by involving part-time addiction workers as consultants. Other team members were trained in motivational interviewing. Ideally, each team would have at least one full-time substance abuse (Monahan et al., 2005) specialist associated with the clinical ward for dually diagnosed patients (DD Ward) which was set up in 2004 as part of the transmural care system. In conjunction with other regions, we have set up a program to train former patients as future co-workers; the first of these started working in 2006.

\section{DISTRICT-ORIENTED TEAMS}

Each FACT team has its own district, which means that the case managers know the community police officers, general practitioners and people in other services better, which enhances collaboration. This has led to improved community integration and case finding. In our area, as in other areas in the Netherlands, we have community integration programs aimed at achieving better relationships between the neighborhood and the psychiatric patients, and at reducing stigma.

To make optimal use of the FACT teams we not only ensure that patients experience our care in a positive way, but also make efforts to find previously unknown cases. Our case managers watch out for people in their neighborhoods who are in need but are not yet formally known to the system. In each region we have a Public Mental Health Coordination Team, which acts as an advice center and safety net. Members of the community, police or landlords may report people who seem to be neglecting themselves or causing a nuisance. Information from the centers or directly from members of the community leads to assertive outreach: the case managers will contact these people and assess whether or not they are in need of long-term care. If they are, the 
FACT team will start caring for them. The Public Mental Health Coordination Teams have reported that there are fewer problems with mentally ill people and that it has become easier to pass these patients on to our FACT teams. This case-finding practice and improved linking of patients to the care system have led to an increased number of patients in care in the North Holland region (about 200 added clients in a population of 225,000 , i.e. $20 \%$ more); as a result, a fifth team has been set up in 2006 .

\section{CONTINUITY OF CARE}

Continuity of care is an important factor for mental health care services in linking their patients to the system (Adair et al., 2005; McGrew, Pescosolido, \& Wright, 2003). It is supported by the 'transmural care' system described above (see under heading Mental health care in the Netherlands). FACT teams work in conjunction with a specialized emergency ward for long-term patients and with the DD ward. Psychiatrists in the FACT teams and in the wards and day clinics work together closely and have weekly meetings. Continuity of care is assured by a shared vision and organization, by the use of transmural treatment plans, and on a personal level by the case managers, who visit their clients in the hospital ward and make arrangements for their discharge.

Continuity of care has improved, also in situations in which ACT or readmission is needed. It contributes to achieving goals relating to the rehabilitation and recovery of patients. The FACT teams meet the requirements which van Weeghel et al. (2005) found to be most important: trusting relationships, individual treatment tailoring and accessibility.

\section{FUTURE DIRECTIONS}

By 2007 we hope to have 9 FACT teams to cover a region of 425,000 inhabitants. Our model has been disseminated to other regions in the Netherlands and FACT seems to be developing into a standard model, which is used more frequently than ACT. The development of the FACT model was prompted by certain characteristics of rural communities. At first it seemed that ACT would be the model of choice in the larger cities, but the city of Amsterdam (900,000 inhabitants) is also consid- 
ering setting up 16 FACT teams and another 3 ACT teams in 2007. There is a preference for FACT as the basic structure for long-term care and treatment in the urban neighborhoods because the expectation is that for the majority group FACT teams will provide more continuity of care and more opportunities for integration into the neighborhood. On the other hand, a number of ACT teams will be needed to provide assertive follow-up throughout the city for homeless people or people who move frequently from one neighborhood to another.

Considerable discussion has taken place on the topic of whether ACT, which is an evidence-based program, can be replaced by FACT, which has not been formally evaluated. Several arguments seem to support our approach. Firstly, ACT has not been researched in the Netherlands, and therefore it is not clear whether the findings of studies in the U.S. would be confirmed in Dutch studies. Our mental health care system is richer and more solid: for example, we have good, accessible 24-hour services, reasonably good housing and sufficient hospital beds. Secondly, the debate in England about the relative merits of ACT and Community Mental Health Teams (CMHT) provides arguments in our favor (Burns, Fioritti, Holloway, Malm, \& Rössler, 2001). English ACT teams did not perform better than the CMHTs, which have many aspects in common with FACT. The Netherlands Institute of Mental Health and Addiction, in collaboration with 20 ACT and FACT teams, has now set up a study focusing on the relationship between fidelity to the ACT model, quality of implementation and outcomes for patients. Recently Bak and others (2007) published first findings of an observational, "real life", pre-post comparison of the introduction of FACT in a region in the south of the Netherlands. Their results are encouraging. The proportion of patients that the transition to remission increased from $19 \%$ in the period before the introduction of FACT, to $31 \%$ in the period with FACT $(\mathrm{OR}=2.21,95 \%$ CI 1.03-4.78). They conclude that FACT may bring improvement to the lives of patients living in countries characterised by fragmented and hospital-based mental health services.

Another issue is the appropriate capacity for ACT teams; our experience leads us to challenge the $20 \%$ rule of thumb that has appeared in the literature. This percentage is arbitrary and not empirically based. One of the potential contributions of the FACT model (if correctly implemented) is to determine the size of the ACT team truly according to need, and not on the basis of some arbitrary percentage. In our project we found after 2 years that approximately $80 \%$ of patients known to a FACT team had been on the whiteboard at some point. This 
demonstrates the vital importance of the continuity of care achieved by working with one team.

The implementation of the FACT teams was supported by the financial system, which has operated in the past. Until now the whole 'transmural' mental health care system has been financed under one Act. However, changes in other sectors (nursing homes, general home care and general hospitals) and rising expenditure for health care as a whole have led the Government to introduce a new financial system, which will be implemented during the coming years. As a result, various components of the service provided by FACT teams and the transmural circuit will be financed from several different sources and under different legislation. Insurance companies, municipalities and possibly the Minister of Justice will be the new financial stakeholders. There is a real threat of fragmentation of FACT and other psychiatric services.

\section{CONCLUSION}

Assertive Community Treatment has been an inspiration to many clinicians in the world. One of the most valuable contributions of the ACT model is that it shifts the focus of treatment from the clinic to the community. The assertive outreach component has proved to be particularly effective in averting relapses, rehospitalizations, and other crises. In the Netherlands it has only been in the last 5 years that it has started to be adopted as a serious model. The Dutch system was more hospital-based, with a 24-hour emergency system, which works reasonably well, but a fragmented system for long-term psychiatric patients in the community. Since 2000 the community and the politicians have been asking for better psychiatric services; ACT was seen as a possible solution. In the Dutch system a new version was developed: the FACT model, or Flexible Assertive Community Treatment. This model, which was first introduced in our region, is now being adopted in the Netherlands on an expanding scale. Some of the advantages offered by the model are continuity of care, treatment and rehabilitation, more opportunities for an individual, recovery-oriented approach, greater possibilities for social integration of the patients because the teams are district-oriented, and the possibility of implementing other evidencebased practices in programs covering all the long-term psychiatric patients in the community. The model is flexible in terms of varying the intensity of service need depending on the clients' needs. FACT seems to be a model, which can address the fragmentation of the Dutch 


\section{system and provide stepped care to all long-term patients outside the hospital.}

\section{REFERENCES}

Adair, C. E. et al. (2005). Continuity of care and health outcomes among persons with severe mental illness. Psychiatric Services, 56, 1061-1069.

Bak, M. et al. (2007). An observational, 'real life' trial of the introduction of Assertive Community Treatment in a geographically defined area using clinical rather than service use outcome criteria. Social Psychiatry Psychiatric Epidemiology, 42, 125-130.

Bond, G.R. et al. (2005). Toekomstige ontwikkelingen van ACT. In C. L. Mulder, \& H. Kroon (Eds.), Assertive community treatment (pp. 205-241). Uitgeverij Cure and Care Publishers, Nijmegen.

Bond, G. R., Drake, R. E., Mueser, K. T., \& Latimer, E. (2001). Assertive community treatment for people with severe mental illness, critical ingredients and impact on patients. Disease Management and Health Outcomes, 9, 142-157.

Bond, G. R., \& Salyers, M. P. (2004). Prediction of outcome from the Dartmouth Assertive Community Treatment Scale. CNS Spectrum, 9, 937-942.

Burns, T., Fioritti, A., Holloway, F., Malm, U., \& Rössler, W. (2001). Case management and assertive community treatment in Europe. Psychiatric Services, 52, 631-636.

Henselmans, H., Kok, S., Nammensma, V., \& van Berkel, H. (1991). Bemoeizorg. Maandblad Geestelijke Volksgezondheid, 46, 1193-1205.

Kluiter, H. (1997). Inpatient treatment and care arrangements to replace or avoid it, searching for an evidence based balance. Current Opinion in Psychiatry, 10, 160-167.

Marshall, M. \& Lockwood, A. (2002). Assertive Community Treatment for people with severe mental disorders. The Cochrane Library, Issue 4.

McGrew, J. H., Pescosolido, B., \& Wright, E. (2003). Case managers' perspectives on critical ingredients of assertive community treatment and on its implementation. Psychiatric Services, $54,370-376$.

Monahan, J. et al. (2005). Use of leverage to improve adherence to psychiatric treatment in the community. Psychiatric Services, 56, 37-44.

Priebe, S. et al. (2005). Reinstitutionalisation in mental health care: Comparison on service provision from six European countries. British Medical Journal, 330, 123-126.

Priebe, S., Huxley, P., Knight, S., \& Evans, S. (1999). Application and results of the Manchester Short Assessment of Quality of Life (MANSA). International Journal of Social Psychiatry, 45, $7-12$.

Ravelli, D. P. (2005). Deinstitutionalisation of mental health care in the Netherlands from 1993-2004. Utrecht.

Stein, L. I. (1990). A historical review of the Madison model of Community Care Assertive Community Treatment. Hospital and Community Psychiatry, 41, 649-651.

Stein, L. I., \& Santos, A. B. (1998). Assertive community. Treatment of persons with severe mental illness. New York.

Teague, G. B., Bond, G. R., \& Drake, R. E. (1998). Program Fidelity in assertive community treatment: Development and use of a measure. American Journal of Orthopsychiatry, 68, 216-232.

Test, L. I., \& Stein, M. A. (Eds.) (1978). Alternatives to mental hospital treatment, USA: Plenum Press.

van Veldhuizen, J. R. (1998). Zorgverlamming. Maandblad Geestelijke volksgezondheid, 53, 1175-1186.

van Veldhuizen, J. R., Wiersma, D., \& Ram, L. M. (1988). Opname Vervangende Dag Behandeling in ontwikkeling; Een bericht uit het Substitutieproject Drenthe. Maandblad Geestelijke Volksgezondheid, 43, 3-18. 
van Weeghel, J. et al. (2005). The components of good community care for people with severe mental illnesses: Views of stakeholders in five European countries. Psychiatric Rehabilitation Journal, 28, 274-281.

Wing, J. K., et al. (1998). Health of the Nation Outcome Scale (HoNOS), research and development. British Journal of Psychiatry, 172, 11-18. 